\title{
BOUNDARY FUNCTIONS AND SETS OF CURVILINEAR CONVERGENCE FOR CONTINUOUS FUNCTIONS
}

\author{
BY

\section{T. J. KACZYNSKI}

Let $D$ be the open unit disk in the complex plane, and let $C$ be its boundary, the unit circle. If $x \in C$, then by an arc at $x$ we mean a simple arc $\gamma$ with one end point at $x$ such that $\gamma-\{x\} \subseteq D$. If $f$ is a function mapping $D$ into some metric space $M$, then the set of curvilinear convergence of $f$ is defined to be

$\{x \in C:$ there exists an $\operatorname{arc} \gamma$ at $x$ and there exists a point

$$
p \in M \text { such that } f(z) \rightarrow p \text { as } z \rightarrow x \text { along } \gamma\} \text {. }
$$

If $\phi$ is a function whose domain is a subset $E$ of the set of curvilinear convergence of $f$, then $\phi$ is called a boundary function for $f$ if, and only if, for each $x \in E$ there exists an arc $\gamma$ at $x$ such that $f(z) \rightarrow \phi(x)$ as $z \rightarrow x$ along $\gamma$. Let $S$ be another metric space. We shall say that a function $\phi$ is of Baire class $\leqq 1(S, M)$ if

(i) domain $\phi=S$,

(ii) range $\phi \subseteq M$, and

(iii) there exists a sequence $\left\{\phi_{n}\right\}$ of continuous functions, each mapping $S$ into $M$, such that $\phi_{n} \rightarrow \phi$ pointwise on $S$.

We shall say that $\phi$ is of honorary Baire class $\leqq 2(S, M)$ if

(i) domain $\phi=S$,

(ii) range $\phi \subseteq M$, and

(iii) there exists a countable set $N \subseteq S$ and there exists a function $\psi$ of Baire class $\leqq 1(S, M)$ such that $\phi(x)=\psi(x)$ for every $x \in S-N$.

It is known that if $f$ is a continuous function mapping $D$ into the Riemann sphere, then the set of curvilinear convergence of $f$ is of type $F_{\sigma \delta}$, and any boundary function for $f$ is of honorary Baire class $\leqq 2(C$, Riemann sphere). (See [3], [4], [5], [6], [9].) J. E. McMillan [6] posed the following problem. If $A$ is a given set in $C$ of type $F_{\sigma \delta}$, and if $\phi$ is a function of honorary Baire class $\leqq 2(A$, Riemann sphere), does there always exist a continuous function $f$ mapping $D$ into the Riemann sphere such that $A$ is the set of curvilinear convergence of $f$ and $\phi$ is a boundary function for $f$ ? The purpose of this paper is to give an affirmative answer to McMillan's question. However, the corresponding question for real-valued functions remains open. (See Problems 1 and 2 at the end of this paper.) In proving our result, we first give a proof under the assumption that $\phi$ is a bounded complexvalued function, and we then use a certain device to transfer the theorem to the Riemann sphere. As we shall indicate in an appendix, the same device can be

Received by the editors September 20, 1968. 
used to transfer certain results concerning real-valued functions of the first Baire class to the case of functions taking values on the Riemann sphere.

Our proof is divided into several major steps, which are labeled (A), (B), (C), etc. The proofs of some of the major steps are divided into smaller steps, which are labeled (I), (II), (III), etc. The results (A) and (B) are taken from the author's doctoral dissertation [5].

Throughout this paper we shall use the following notation. $R$ denotes the set of real numbers, $S^{2}$ denotes the Riemann sphere, and $R^{n}$ denotes $n$-dimensional Euclidean space. Points in $R^{n}$ will be written in the form $\left\langle x_{1}, x_{2}, \ldots, x_{n}\right\rangle$ (rather than $\left.\left(x_{1}, x_{2}, \ldots, x_{n}\right)\right)$ in order to avoid confusion with open intervals of real numbers in the case $n=2$. The empty set will be denoted by $\varnothing$. When we speak of a complex-valued function, we mean a function taking only finite complex values. The closure of a set $E$ will be denoted either by $\bar{E}$ or by $\mathrm{Cl} E$. If $I$ is an interval of real numbers, then $I^{*}$ denotes the interior of $I$. If $p$ is a point of some metric space and $r \in(0,+\infty)$, then $S(r, p)$ denotes the set of all points of the space having distance (strictly) less than $r$ from $p$.

We define

$$
\begin{aligned}
& Q=\left\{\langle x, y\rangle \in R^{2}:-1 \leqq x \leqq 1,0<y \leqq 1\right\} \\
& X=\{\langle x, 0\rangle:-1<x<1\} \\
& H=\left\{\langle x, y\rangle \in R^{2}: y>0\right\}
\end{aligned}
$$

It will be convenient to identify $\langle x, 0\rangle$ with the real number $x$, and $X$ with $(-1,1)$. If $f$ is a complex-valued function defined in $Q$, then we shall understand the set of curvilinear convergence of $f$ to mean the set of all $x \in X$ for which there exists an $\operatorname{arc} \gamma$ at $x$ (contained in the interior of $Q$ except for its end point at $x$ ) such that $f$ approaches a finite limit along $\gamma$. If $a \in X, \varepsilon>0$, and $0<\theta<\frac{1}{2} \pi$, then we let

$$
s(a, \varepsilon, \theta)=\left\{\langle x, y\rangle \in R^{2}: 0<y<\varepsilon, a-y \operatorname{ctn} \theta<x<a+y \operatorname{ctn} \theta\right\} .
$$

Thus $s(a, \varepsilon, \theta)$ is the interior of an isosceles triangle in $H$ with apex at $a$.

(A) If $A \subseteq X$ is a set of type $F_{\sigma \delta}$, then there exists a bounded continuous realvalued function $g$ defined in $Q$ such that

(i) for each $x \in A, g(z) \rightarrow 0$ as $z$ approaches $x$ through $s\left(x, 1, \frac{3}{8} \pi\right)$, and

(ii) if $x \in X$, and if there exists an arc $\gamma$ at $x$ such that $g(z) \rightarrow 0$ as $z$ approaches $x$ along $\gamma$, then $x \in A$.

(I) Let $E_{1}$ and $E_{2}$ be two sets on the real line. A point $p \in R$ will be called a splitting point for $E_{1}$ and $E_{2}$ if either

(i) $x_{1} \leqq p$ for all $x_{1} \in E_{1}$ and $p \leqq x_{2}$ for all $x_{2} \in E_{2}$, or

(ii) $x_{2} \leqq p$ for all $x_{2} \in E_{2}$ and $p \leqq x_{1}$ for all $x_{1} \in E_{1}$.

We will say that $E_{1}$ and $E_{2}$ split if and only if there exists a splitting point for $E_{1}$ and $E_{2}$.

(II) By a special family we mean a family $\mathscr{F}$ of subsets of $X$ such that

(i) $\mathscr{F}$ is nonempty, 
(ii) $\mathscr{F}$ is countable,

(iii) each member of $\mathscr{F}$ is compact,

(iv) if $E, F \in \mathscr{F}$, then either $E=F, E \cap F=\varnothing$, or $E$ and $F$ split.

(III) If $E \subseteq X$ is an $F_{\sigma}$ set, then there exists a special family $\mathscr{F}$ such that $E=\bigcup \mathscr{F}$.

Proof. We can write $E=\bigcup_{n=1}^{\infty} A_{n}$, where $A_{1}=\varnothing, A_{n}$ is closed, and $A_{n} \subseteq A_{n+1}$ for all $n$. Observe that if $I$ is any open interval contained in $X$, then there exists a countable family $\left\{J_{n}\right\}_{n=1}^{\infty}$ of compact intervals contained in $X$ such that $I=\bigcup_{n=1}^{\infty} J_{n}$, and $n \neq m$ implies that $J_{n}$ and $J_{m}$ split. Since $X-A_{n}$ is a countable disjoint union of open intervals, it follows that we can choose (for each $n$ ) a family

$$
\left\{I_{n, j}\right\}_{j=1}^{\infty}
$$

of compact intervals such that $X-A_{n}=\bigcup_{j=1}^{\infty} I_{n, j}$, and $j \neq k$ implies that $I_{n, j}$ and $I_{n, k}$ split. Let

$$
\mathscr{F}=\left\{A_{1}\right\} \cup\left\{I_{n, j} \cap A_{n+1}: n=1,2, \ldots ; j=1,2, \ldots\right\} .
$$

Then $\mathscr{F}$ is a countable family of compact sets, and

$$
\begin{aligned}
E & =\bigcup_{n=1}^{\infty} A_{n}=A_{1} \cup \bigcup_{n=1}^{\infty} A_{n+1} \cap\left(X-A_{n}\right) \\
& =A_{1} \cup \bigcup_{n=1}^{\infty} \bigcup_{j=1}^{\infty} A_{n+1} \cap I_{n, j} \\
& =\bigcup \mathscr{F} .
\end{aligned}
$$

Let $F_{1}$ and $F_{2}$ be any two distinct members of $\mathscr{F}$. If either $F_{1}$ or $F_{2}$ is $A_{1}=\varnothing$, then $F_{1}$ and $F_{2}$ are automatically disjoint. If neither $F_{1}$ nor $F_{2}$ is $A_{1}$, then we can write

$$
\begin{aligned}
& F_{1}=I_{n(1), j(1)} \cap A_{n(1)+1}, \\
& F_{2}=I_{n(2), j(2)} \cap A_{n(2)+1} .
\end{aligned}
$$

If $n(1)<n(2)$, then $n(1)+1 \leqq n(2)$, so

$$
F_{2}=I_{n(2), j(2)} \cap A_{n(2)+1} \subseteq X-A_{n(2)} \subseteq X-A_{n(1)+1} \subseteq X-F_{1},
$$

and therefore $F_{1}$ and $F_{2}$ are disjoint. If $n(2)<n(1)$, a similar argument shows that $F_{1}$ and $F_{2}$ are disjoint. Now suppose $n(1)=n(2)$. Then, since $F_{1} \neq F_{2}$, we have $j(1) \neq j(2)$. So $I_{n(1), j(2)}=I_{n(2), j(2)}$ and $I_{n(1), j(1)}$ split, and consequently $F_{1}$ and $F_{2}$ split. We have shown that any two distinct members of $\mathscr{F}$ either split or are disjoint, so $\mathscr{F}$ is a special family.

(IV) Let $A \subseteq X$ be a set of type $F_{\sigma \delta}$. Then there exists a sequence of special families $\left\{\mathscr{F}_{n}\right\}_{n=1}^{\infty}$ such that

(i) $A=\bigcap_{n=1}^{\infty}\left(\bigcup \mathscr{F}_{n}\right)$,

(ii) if $n \geqq 1$ and $E \in \mathscr{F}_{n+1}$, then there exists $F \in \mathscr{F}_{n}$ with $E \subseteq F$.

Proof. There exist $F_{\sigma}$ sets $A_{1} \supseteq A_{2} \supseteq A_{3} \supseteq \cdots$ such that $A=\bigcap_{n=1}^{\infty} A_{n}$. By (III), we can choose (for each $n$ ) a special family $\mathscr{E}_{n}$ such that $A_{n}=\bigcup \mathscr{E}_{n}$. Let $\mathscr{F}_{1}=\mathscr{E}_{1}$. For $n \geqq 1$, let

$$
\mathscr{F}_{n+1}=\left\{F \cap E: F \in \mathscr{F}_{n} \text { and } E \in \mathscr{E}_{n+1}\right\}
$$


By induction on $n$, one can show that each $\mathscr{F}_{n}$ is a special family and that $A_{n}=\bigcup \mathscr{F}_{n}$. It is clear that the other conditions are satisfied.

(V) Suppose that $J$ is a nonempty interval with $\bar{J} \subseteq X$, and let $a, b(a \leqq b)$ be the end points of $J$. By Trap $(J, \varepsilon, \theta)$ (where $\theta \in\left(0, \frac{1}{2} \pi\right)$ and $\left.\varepsilon>0\right)$ we mean the trapezoidshaped open set defined by

$$
\operatorname{Trap}(J, \varepsilon, \theta)=\{\langle x, y\rangle: 0<y<\varepsilon, a+y \operatorname{ctn} \theta<x<b-y \operatorname{ctn} \theta\} .
$$

For $\theta \in\left(0, \frac{1}{2} \pi\right)$ let Tri $(J, \theta)$ be the closed triangular area defined by

$$
\operatorname{Tri}(J, \theta)=\{\langle x, y\rangle: y \geqq 0, a+y \operatorname{ctn} \theta \leqq x \leqq b-y \operatorname{ctn} \theta\} .
$$

If $K$ is a nonempty compact subset of $X$, let $J(K)$ be the smallest closed interval containing $K$. If $\varepsilon>0$ and $0<\beta<\alpha<\frac{1}{2} \pi$, then we define

$$
B(K, \varepsilon, \alpha, \beta)=\operatorname{Trap}(J(K), \varepsilon, \alpha)-\bigcup_{I \in \mathscr{I}} \operatorname{Tri}(I, \beta),
$$

where $\mathscr{I}$ denotes the (possibly empty) set of disjoint nonempty open intervals whose union is $J(K)-K$.

We state without proof the following readily verifiable facts ((VI) through (XVIII)).

(VI) $s(x, \varepsilon, \theta)$ is an open subset of $H$.

(VII) $\mathrm{Cl}[s(x, \varepsilon, \theta)] \cap X=\{x\}$.

(VIII) If $\varepsilon<\varepsilon^{\prime}$ and $\theta^{\prime}<\theta$, then $\mathrm{Cl}[s(x, \varepsilon, \theta)] \cap H \subseteq s\left(x, \varepsilon^{\prime}, \theta^{\prime}\right)$.

(IX) If $x \neq y$ and $\varepsilon, \theta$ are given, then there exists $\delta>0$ such that, for every $\eta \leqq \delta, s(x, \varepsilon, \theta)$ and $s(y, \eta, \theta)$ are disjoint.

(X) $B(K, \varepsilon, \alpha, \beta)$ is an open subset of $H$.

(XI) If $K_{1}$ and $K_{2}$ split, then, for any $\varepsilon_{1}, \varepsilon_{2}, \alpha$, and $\beta, B\left(K_{1}, \varepsilon_{1}, \alpha, \beta\right)$ and $B\left(K_{2}, \varepsilon_{2}, \alpha, \beta\right)$ are disjoint.

(XII) If $K_{1}$ and $K_{2}$ are disjoint compact subsets of $X$, and if $\varepsilon, \alpha, \beta$ are given, then there exists $\delta>0$ such that for every $\eta \leqq \delta, B\left(K_{1}, \varepsilon, \alpha, \beta\right)$ and $B\left(K_{2}, \eta, \alpha, \beta\right)$ are disjoint.

(XIII) $\mathrm{Cl}[B(K, \varepsilon, \alpha, \beta)] \cap X \subseteq K$.

(XIV) Suppose that $K_{1} \subseteq K, \varepsilon>\varepsilon_{1}>0$, and $0<\beta<\beta_{1}<\alpha_{1}<\alpha<\pi / 2$. Then $\mathrm{Cl}\left[B\left(K_{1}, \varepsilon_{1}, \alpha_{1}, \beta_{1}\right)\right] \cap H \subseteq B(K, \varepsilon, \alpha, \beta)$.

(XV) Suppose that $\alpha<\theta<\frac{1}{2} \pi$ and $x \notin J(K)^{*}$. Then, for any $\varepsilon, \varepsilon_{1}$, and $\beta$, $B(K, \varepsilon, \alpha, \beta)$ and $s\left(x, \varepsilon_{1}, \theta\right)$ are disjoint.

(XVI) Suppose that $x \notin K$ and that $\varepsilon, \alpha, \beta, \theta$ are given. Then there exists $\delta>0$ such that for every $\eta \leqq \delta, s(x, \eta, \theta)$ and $B(K, \varepsilon, \alpha, \beta)$ are disjoint.

(XVII) Suppose that $x \notin K$ and that $\delta, \alpha, \beta, \theta$ are given. Then there exists $\varepsilon>0$ such that for every $\eta \leqq \varepsilon, s(x, \delta, \theta)$ and $B(K, \eta, \alpha, \beta)$ are disjoint.

(XVIII) Suppose that $x \in K \cap J(K)^{*}$ and $0<\beta<\alpha<\theta<\frac{1}{2} \pi$. Let $\varepsilon$ be given. Then there exists $\delta>0$ such that for every $\eta \leqq \delta, \mathrm{Cl}[s(x, \eta, \theta)] \cap H \subseteq B(K, \varepsilon, \alpha, \beta)$.

(XIX) If $\mathscr{F}$ is a special family, let $\mathscr{F}^{2}$ be the set of all members of $\mathscr{F}$ that have two or more points, and let $E(\mathscr{F})$ be the set of all end points of intervals $J(F)$, where $F \in \mathscr{F}$ and $F \neq \varnothing$. 
Suppose that $0<\beta<\alpha<\theta<\frac{1}{2} \pi$, and that $\mathscr{F}$ is a special family. By a pair of special $\alpha, \beta, \theta$ functions for $\mathscr{F}$, I mean a pair $(\varepsilon, \delta)$, where $\varepsilon$ and $\delta$ are positive real-valued functions, the domain of $\varepsilon$ is $E(\mathscr{F})$, the domain of $\delta$ is $\mathscr{F}^{2}$, and

(i) for each $\eta>0$, there exist at most finitely many $F \in \mathscr{F}^{2}$ such that $\delta(F) \geqq \eta$;

(ii) for each $\eta>0$, there exist at most finitely many $x \in E(\mathscr{F})$ such that $\varepsilon(x) \geqq \eta$;

(iii) if $x, x^{\prime} \in E(\mathscr{F})$ and $x \neq x^{\prime}$, then $s(x, \varepsilon(x), \theta)$ and $s\left(x^{\prime}, \varepsilon\left(x^{\prime}\right), \theta\right)$ are disjoint;

(iv) if $F, K \in \mathscr{F}^{2}$ and $F \neq K$, then $B(F, \delta(F), \alpha, \beta)$ and $B(K, \delta(K), \alpha, \beta)$ are disjoint;

(v) if $x \in E(\mathscr{F})$ and $F \in \mathscr{F}^{2}$, then $s(x, \varepsilon(x), \theta)$ and $B(F, \delta(F), \alpha, \beta)$ are disjoint.

(XX) Let $\mathscr{F}$ be a special family and suppose that $0<\beta<\alpha<\theta<\frac{1}{2} \pi$. Then there exists a pair of special $\alpha, \beta, \theta$ functions for $\mathscr{F}$.

Though a formal proof of this statement is lengthy, it requires no originality, so we omit the details. The idea is to arrange the members of $\mathscr{F}$ in a finite or infinite sequence $F_{1}, F_{2}, F_{3}, \ldots$, and then define $\varepsilon$ and $\delta$ inductively. One makes use of statements (IX), (XI), (XII), (XV), (XVI), (XVII).

(XXI) Let $\mathscr{F}$ be a special family, and suppose $0<\beta<\alpha<\theta<\frac{1}{2} \pi$. Let $(\varepsilon, \delta)$ be a pair of special $\alpha, \beta, \theta$ functions for $\mathscr{F}$. If $\varepsilon_{1}, \delta_{1}$ are two real-valued functions having domains $E(\mathscr{F})$ and $\mathscr{F}^{2}$ repectively, and if

$$
\begin{array}{ll}
0<\varepsilon_{1}(x) \leqq \varepsilon(x) & \text { for all } x \in E(\mathscr{F}), \\
0<\delta_{1}(F) \leqq \delta(F) & \text { for all } F \in \mathscr{F}^{2},
\end{array}
$$

then $\left(\varepsilon_{1}, \delta_{1}\right)$ is a pair of special $\alpha, \beta, \theta$ functions for $\mathscr{F}$.

The proof of this statement is trivial.

(XXII) We now proceed to the proof of statement (A) itself. Let $A$ be our given $F_{\sigma \delta}$ set. By (IV), we can choose a sequence of special families $\left\{\mathscr{F}_{n}\right\}_{n=1}^{\infty}$ such that $A=\bigcap_{n=1}^{\infty}\left(\bigcup \mathscr{F}_{n}\right)$, and for each $K \in \mathscr{F}_{n+1}$ there exists $F \in \mathscr{F}_{n}$ with $K \subseteq F$.

Let $\left\{\beta_{n}\right\}_{n=1}^{\infty}$ be a strictly increasing sequence in $\left(0, \frac{1}{8} \pi\right)$ coverging to $\frac{1}{8} \pi$.

Let $\left\{\alpha_{n}\right\}_{n=1}^{\infty}$ be a strictly decreasing sequence in $\left(\frac{1}{8} \pi, \frac{1}{4} \pi\right)$ converging to $\frac{1}{8} \pi$.

Let $\left\{\theta_{n}\right\}_{n=1}^{\infty}$ be a strictly increasing sequence in $\left(\frac{1}{4} \pi, \frac{3}{8} \pi\right)$ converging to $\frac{3}{8} \pi$.

Let $E_{n}=E\left(\mathscr{F}_{n}\right)$.

Let $(\varepsilon(1, \cdot), \delta(1, \cdot))$ be any pair of special $\alpha_{1}, \beta_{1}, \theta_{1}$ functions for $\mathscr{F}_{1}$.

Now suppose that for each $k \leqq n$ we have chosen a pair of special $\alpha_{k}, \beta_{k}, \theta_{k}$ functions $(\varepsilon(k, \cdot), \delta(k, \cdot))$ for $\mathscr{F}_{k}$ in such a way that

(i) whenever $1 \leqq k \leqq n-1, x \in E_{k+1}, F \in \mathscr{F}_{k}$, and $x \in F \cap J(F)^{*}$, then

$$
\mathrm{Cl}\left[s\left(x, \varepsilon(k+1, x), \theta_{k+1}\right)\right] \cap H \subseteq B\left(F, \delta(k, F), \alpha_{k}, \beta_{k}\right) ;
$$

(ii) whenever $1 \leqq k \leqq n-1, x \in E_{k+1}$, and $x \in E_{k}$, then

$$
\mathrm{Cl}\left[s\left(x, \varepsilon(k+1, x), \theta_{k+1}\right)\right] \cap H \subseteq s\left(x, \varepsilon(k, x), \theta_{k}\right) ;
$$

(iii) whenever $1 \leqq k \leqq n-1, K \in\left(\mathscr{F}_{k+1}\right)^{2}, F \in\left(\mathscr{F}_{k}\right)^{2}$, and $K \subseteq F$, then

$$
\mathrm{Cl}\left[B\left(K, \delta(k+1, K), \alpha_{k+1}, \beta_{k+1}\right)\right] \cap H \subseteq B\left(F, \delta(k, F), \alpha_{k}, \beta_{k}\right) .
$$


Then we construct $(\varepsilon(n+1, \cdot), \delta(n+1, \cdot))$ as follows. Let $(\varepsilon, \delta)$ be any pair of special $\alpha_{n+1}, \beta_{n+1}, \theta_{n+1}$ functions for $\mathscr{F}_{n+1}$. If $x \in E_{n+1}-E_{n}$, then for some unique $F \in\left(\mathscr{F}_{n}\right)^{2}, x \in F \cap J(F)^{*}$. By (XVIII), we can choose $\xi(x)>0$ so that $\eta \leqq \xi(x)$ implies

$$
\mathrm{Cl}\left[s\left(x, \eta, \theta_{n+1}\right)\right] \cap H \subseteq B\left(F, \delta(n, F), \alpha_{n}, \beta_{n}\right) .
$$

We set $\varepsilon(n+1, x)=\min \{\varepsilon(x), \xi(x)\}$. On the other hand, if $x \in E_{n+1} \cap E_{n}$, then we set $\varepsilon(n+1, x)=\min \left\{\varepsilon(x), \frac{1}{2} \varepsilon(n, x)\right\}$.

If $K \in\left(\mathscr{F}_{n+1}\right)^{2}$, then there exists a unique $F \in\left(\mathscr{F}_{n}\right)^{2}$ with $K \subseteq F$. Set

$$
\delta(n+1, K)=\min \left\{\delta(K), \frac{1}{2} \delta(n, F)\right\} .
$$

By (XXI), $(\varepsilon(n+1, \cdot), \delta(n+1, \cdot))$ is a pair of special $\alpha_{n+1}, \beta_{n+1}, \theta_{n+1}$ functions for $\mathscr{F}_{n+1}$, and, by (VIII) and (XIV), conditions (i), (ii), (iii) are still satisfied when $n$ is replaced by $n+1$. Thus we can inductively construct a pair $(\varepsilon(n, \cdot), \delta(n, \cdot))$ of special $\alpha_{n}, \beta_{n}, \theta_{n}$ functions for $\mathscr{F}_{n}$ in such a way that (i), (ii), and (iii) are satisfied for every $n$.

Let

$$
U_{n}=\left[\bigcup_{x \in E_{n}} s\left(x, \varepsilon(n, x), \theta_{n}\right)\right] \cup\left[\bigcup_{F \in\left(\mathscr{F}_{n}\right)^{2}} B\left(F, \delta(n, F), \alpha_{n}, \beta_{n}\right)\right]
$$

Then $U_{n}$ is open. For fixed $n$, all the various sets $s\left(x, \varepsilon(n, x), \theta_{n}\right)\left(x \in E_{n}\right)$ and $B\left(F, \delta(n, F), \alpha_{n}, \beta_{n}\right)\left(F \in\left(\mathscr{F}_{n}\right)^{2}\right)$ are open and pairwise disjoint, so that every component of $U_{n}$ is contained in one of the sets $s\left(x, \varepsilon(n, x), \theta_{n}\right)\left(x \in E_{n}\right)$ or $B\left(F, \delta(n, F), \alpha_{n}, \beta_{n}\right)\left(F \in\left(\mathscr{F}_{n}\right)^{2}\right)$. It therefore follows from (VII) and (XIII) that if $W$ is any component of $U_{n}$, then

$$
\bar{W} \cap X \subseteq \cup \mathscr{F}_{n} .
$$

From conditions (i) and (ii) in the definition of a pair of special $\alpha, \beta, \theta$ functions, it follows that

$$
\bar{U}_{n} \cap H=\left[\bigcup_{x \in E_{n}} \mathrm{Cl}\left[s\left(x, \varepsilon(n, x), \theta_{n}\right)\right] \cap H\right] \cup\left[\bigcup_{F \in\left(\mathscr{F}_{n}\right)^{2}} \mathrm{Cl}\left[B\left(F, \delta(n, F), \alpha_{n}, \beta_{n}\right)\right] \cap H\right] .
$$

Consequently, conditions (i), (ii), (iii) in our inductive construction of $(\varepsilon(n, \cdot)$, $\delta(n, \cdot)$ ) (together with the fact that $x \in E_{n+1}-E_{n}$ implies $x \in F \cap J(F)^{*}$ for some $\left.F \in\left(\mathscr{F}_{n}\right)^{2}\right)$ imply that $\bar{U}_{n+1} \cap H \subseteq U_{n}$ for every $n$.

By Urysohn's Lemma there exists a continuous function $g_{n}: H \rightarrow[0,1]$ such that $g_{n}(z)=1$ for $z \in H-U_{n}$ and $g_{n}(z)=0$ for $z \in \bar{U}_{n+1} \cap H$. Let

$$
g(z)=\sum_{n=1}^{\infty}\left(\frac{1}{2}\right)^{n} g_{n}(z)
$$

Then $0 \leqq g(z) \leqq 1$, and the series converges uniformly, so $g$ is continuous in $H$.

If $z \in H-U_{n}$, then $z \in H-U_{m}$ for every $m \geqq n$, so that $1=g_{n}(z)=g_{n+1}(z)=g_{n+2}(z)$ $=\cdots$, and hence

$$
g(z) \geqq \sum_{m=n}^{\infty}\left(\frac{1}{2}\right)^{m}=\left(\frac{1}{2}\right)^{n-1} \quad\left(z \in H-U_{n}\right) .
$$


Also, if $z \in U_{n+1}$, then $z \in U_{1}, U_{2}, \ldots, U_{n+1}$, so that $0=g_{1}(z)=g_{2}(z)=\cdots=g_{n}(z)$, and

$$
g(z) \leqq \sum_{m=n+1}^{\infty}\left(\frac{1}{2}\right)^{m}=\left(\frac{1}{2}\right)^{n} \quad\left(z \in U_{n+1}\right)
$$

Let $x_{0} \in A$ be given. We must show that $g(z) \rightarrow 0$ as $z$ approaches $x_{0}$ through $s\left(x_{0}, 1, \frac{3}{8} \pi\right)$. Take any natural number $n$. Since $x_{0} \in \cup \mathscr{F}_{n+1}$, it follows that either $x_{0} \in E_{n+1}$ or else $x_{0} \in F \cap J(F)^{*}$ for some $F \in\left(\mathscr{F}_{n+1}\right)^{2}$. In the first case, set $\eta=\varepsilon\left(n+1, x_{0}\right)$. In the second case, (XVIII) shows that we can choose $\eta>0$ small enough so that

$$
s\left(x_{0}, \eta, \frac{3}{8} \pi\right) \subseteq B\left(F, \delta(n+1, F), \alpha_{n+1}, \beta_{n+1}\right) .
$$

Suppose $\langle x, y\rangle \in s\left(x_{0}, 1, \frac{3}{8} \pi\right)$ and $y<\eta$. Then, in the first case,

$$
\langle x, y\rangle \in s\left(x_{0}, \eta, \frac{3}{8} \pi\right) \subseteq s\left(x_{0}, \varepsilon\left(n+1, x_{0}\right), \theta_{n+1}\right) \subseteq U_{n+1},
$$

and, in the second case,

$$
\langle x, y\rangle \in s\left(x_{0}, \eta, \frac{3}{8} \pi\right) \subseteq B\left(F, \delta(n+1, F), \alpha_{n+1}, \beta_{n+1}\right) \subseteq U_{n+1} .
$$

Thus, referring to (3), we see that $g(x, y) \leqq\left(\frac{1}{2}\right)^{n}$ whenever $\langle x, y\rangle \in s\left(x_{0}, 1, \frac{3}{8} \pi\right)$ and $y<\eta$. Therefore $g(z) \rightarrow 0$ as $z$ approaches $x_{0}$ through $s\left(x_{0}, 1, \frac{3}{8} \pi\right)$.

Let $x_{1}$ be a point of $X$, and assume there exists an arc $\gamma$ at $x_{1}$ such that $g(z) \rightarrow 0$ as $z$ approaches $x_{1}$ along $\gamma$. Then $\gamma$ has a subarc $\gamma^{\prime}$ with one end point at $x_{1}$ such that $\gamma^{\prime}-\left\{x_{1}\right\} \subseteq g^{-1}\left(\left(-\left(\frac{1}{2}\right)^{n},\left(\frac{1}{2}\right)^{n}\right)\right)$. By (2), $\gamma^{\prime}-\left\{x_{1}\right\} \subseteq U_{n}$. Therefore, by (1), $x_{1} \in$ $\cup \mathscr{F}_{n}$. Since $n$ is arbitrary,

$$
x_{1} \in \bigcap_{n=1}^{\infty}\left(\bigcup \mathscr{F}_{n}\right)=A .
$$

Thus, by restricting $g$ to $Q$ we obtain the desired function.

(B) Let $A$ be a subset of $X$ of type $F_{\sigma \delta}$, and let $\phi$ be a bounded complex-valued function of honorary Baire class $\leqq 2\left(A, R^{2}\right)$. Then there exists a bounded continuous complex-valued function $h$ defined in $Q$ such that, for each $x \in A$, there exists an arc $\gamma$ at $x$ with $\gamma-\{x\} \subseteq s\left(x, 1, \frac{3}{8} \pi\right)$ and

$$
\lim _{z \rightarrow x ; z \in \gamma} h(z)=\phi(x)
$$

(I) Let $I$ be a bounded open interval in $R$, and let $f: I \rightarrow R$ be a bounded, strictly increasing function. Then there exists a continuous, weakly increasing function $f^{*}: R \rightarrow R$ such that $f^{*}(f(x))=x$ for every $x \in I$. (This result is probably not new, but I do not know of a reference for it, so I am obliged to prove it here.)

Proof. Let $Z=f(I)$, let $c=\inf Z$, and let $d=\sup Z$. Observe that $Z \subseteq(c, d)$, and that $f^{-1}: Z \rightarrow I$ is strictly increasing. I assert that for each $x \in(c, d)$

$$
\sup f^{-1}((c, x] \cap Z)=\sup f^{-1}((c, x) \cap Z) .
$$

If $x \notin Z$, the equation is trivial. Suppose $x \in Z$. Then

$$
c<y<f^{-1}(x) \Rightarrow(f(y)<x \text { and } f(y) \in Z),
$$


so that $\left(c, f^{-1}(x)\right) \subseteq f^{-1}((c, x) \cap Z)$. Hence

$$
\sup f^{-1}((c, x) \cap Z) \geqq f^{-1}(x)=\sup f^{-1}((c, x] \cap Z) .
$$

The opposite inequality is trivial, so (4) is established.

I also assert that for each $x \in(c, d)$

$$
\inf f^{-1}((x, d) \cap Z)=\sup f^{-1}((c, x] \cap Z) .
$$

Obviously,

$$
\inf f^{-1}((x, d) \cap Z) \geqq \sup f^{-1}((c, x] \cap Z) .
$$

Take any $y>\sup f^{-1}((c, x] \cap Z)$. If $f(y) \leqq x$, then $f(y) \in(c, x] \cap Z$, and so $y \in f^{-1}((c, x] \cap Z)$, a contradiction. Thus $f(y)>x$ and $f(y) \in(x, d) \cap Z$. Therefore $y \in f^{-1}((x, d) \cap Z)$, so that $\inf f^{-1}((x, d) \cap Z) \leqq y$. In view of the choice of $y$, this implies that

$$
\inf f^{-1}((x, d) \cap Z) \leqq \sup f^{-1}((c, x] \cap Z),
$$

and (5) is established.

Define $f^{*}$ on $(c, d)$ by

$$
f^{*}(x)=\sup f^{-1}((c, x] \cap Z) \quad(x \in(c, d)) .
$$

It is clear that $f^{*}$ is weakly increasing and that $f^{*}(f(x))=x$ for every $x \in I$. The continuity of $f^{*}$ can easily be deduced from the equations

$$
\sup f^{*}((c, x))=f^{*}(x), \quad \inf f^{*}((x, d))=f^{*}(x),
$$

which are established as follows:

$$
\begin{aligned}
\sup f^{*}((c, x)) & =\sup _{c<y<x} \sup f^{-1}((c, y] \cap Z) \\
& =\sup f^{-1}((c, x) \cap Z) \\
& =\sup f^{-1}((c, x] \cap Z) \\
& =f^{*}(x), \\
\inf f^{*}((x, d)) & =\inf _{x<y<d} \sup f^{-1}((c, y] \cap Z) \\
& =\inf _{x<y<d} \inf f^{-1}((y, d) \cap Z) \\
& =\inf f^{-1}((x, d) \cap Z) \\
& =\sup f^{-1}((c, x] \cap Z) \\
& =f^{*}(x) .
\end{aligned}
$$

We now extend $f^{*}$ to all of $R$ by setting

$$
\begin{array}{ll}
f^{*}(x)=\inf f^{*}((c, d)) & \text { if } x \leqq c, \\
f^{*}(x)=\sup f^{*}((c, d)) & \text { if } x \geqq d,
\end{array}
$$

and we are finished. 
(II) Suppose that $M$ is a metric space and that $u: M \rightarrow R$ is a function having the following property. For every sequence $\left\{p_{n}\right\}$ of points of $M$, every $p \in M$, and every $y \in R \cup\{-\infty,+\infty\}$, if $p_{n} \rightarrow p$ and $u\left(p_{n}\right) \rightarrow y$ as $n \rightarrow \infty$, then $y \in R$ and $u(p)=y$. Under this hypothesis, $u$ is continuous.

Proof. Let $\left\{p_{n}\right\}$ be any sequence of points in $M$ converging to a point $p \in M$. We have only to show that $u\left(p_{n}\right) \rightarrow u(p)$. But suppose $u\left(p_{n}\right)+u(p)$. Then there exists a subsequence $\left\{u\left(p_{n(k)}\right)\right\}$ and there exists $y \in R \cup\{-\infty,+\infty\}$ such that $y \neq u(p)$ and $u\left(p_{n(k)}\right) \rightarrow y$ as $k \rightarrow \infty$. Since $p_{n(k)} \rightarrow p$ as $k \rightarrow \infty$, this contradicts our hypothesis.

(III) Let $A \subseteq(-1,1)$ be of type $F_{\sigma \delta}$, and let $\psi$ be a complex-valued function of Baire class $\leqq 1\left(A, R^{2}\right)$. Then there exists a sequence $\left\{g_{n}\right\}$ of continuous functions, each mapping $R$ into $R^{2}$, such that for each $x \in A, g_{n}(x) \rightarrow \psi(x)$ as $n \rightarrow \infty$.

Proof. This can be proved in a more general context, as shown in [5]. For a quick proof of the special case stated above, we can refer to a theorem of Bagemihl and McMillan.[1, Theorem 2], which tells us that there exist continuous real-valued functions $f_{1}$ and $f_{2}$ defined in $H$ such that, for each $x \in A, f_{1}$ has angular limit $\operatorname{Re}(\psi(x))$ at $x$ and $f_{2}$ has angular $\operatorname{limit} \operatorname{Im}(\psi(x))$ at $x$. For each $x \in R$, set

$$
g_{n}(x)=f_{1}\left(x, \frac{1}{n}\right)+i f_{2}\left(x, \frac{1}{n}\right)
$$

(IV) Now we proceed to the proof of statement (B). Let $\psi$ be a function of Baire class $\leqq 1\left(A, R^{2}\right)$ and let $E$ be a (possibly empty) countable subset of $A$ such that $\phi(x)=\psi(x)$ for each $x \in A-E$. Let $N$ be an infinite countable set with $E \subseteq N \subseteq X$. Let $w$ be a real-valued function defined on $N$ such that $w(s)>0$ for each $s \in N$ and

$$
\sum_{s \in N} w(s)<2^{1 / 2}-1
$$

For each $x \in X=(-1,1)$, let $N(x)=\{s \in N:-1<s<x\}$. Define $f$ on $(-1,1)$ by setting

$$
f(x)=x+\sum_{s \in N(x)} w(s)
$$

Then $f$ is a bounded, strictly increasing function on $(-1,1)$, and $|f(x)-x|<2^{1 / 2}-1$. By (I), there exists a continuous, weakly increasing function $f^{*}: R \rightarrow R$ such that $f^{*}(f(x))=x$ for each $x \in(-1,1)$.

Let

$$
H_{0}=\left\{\langle x, y\rangle \in R^{2}: 0<y \leqq \frac{1}{2}\right\}
$$

For fixed $\langle x, y\rangle \in H_{0}$,

$$
u-f^{*}\left(\frac{x-(1-y) u}{y}\right)
$$

is a strictly increasing continuous function of $u$ that approaches $+\infty$ as $u \rightarrow+\infty$ 
and $-\infty$ as $u \rightarrow-\infty$. Consequently there exists precisely one number $u(x, y)$ that satisfies the equation

$$
u(x, y)-f^{*}\left(\frac{x-(1-y) u(x, y)}{y}\right)=0 .
$$

I assert that $u(x, y)$ is a continuous function on $H_{0}$. We show this by using (II). Suppose that $\langle x, y\rangle \in H_{0}, u_{0} \in R \cup\{-\infty,+\infty\},\left\{\left\langle x_{n}, y_{n}\right\rangle\right\} \subseteq H_{0},\left\langle x_{n}, y_{n}\right\rangle \rightarrow\langle x, y\rangle$, and $u\left(x_{n}, y_{n}\right) \rightarrow u_{0}$. If $u_{0}=+\infty$, then, as $n \rightarrow \infty$,

and so

$$
\frac{x_{n}-\left(1-y_{n}\right) u\left(x_{n}, y_{n}\right)}{y_{n}} \rightarrow-\infty \text {, }
$$

$$
u\left(x_{n}, y_{n}\right)-f^{*}\left(\frac{x_{n}-\left(1-y_{n}\right) u\left(x_{n}, y_{n}\right)}{y_{n}}\right) \rightarrow+\infty,
$$

which contradicts (6). So $u_{0} \neq+\infty$, and a similar argument shows that $u_{0} \neq-\infty$. Thus, by (6),

$$
\begin{aligned}
0 & =\lim _{n \rightarrow \infty}\left[u\left(x_{n}, y_{n}\right)-f^{*}\left(\frac{x_{n}-\left(1-y_{n}\right) u\left(x_{n}, y_{n}\right)}{y_{n}}\right)\right] \\
& =u_{0}-f^{*}\left(\frac{x-(1-y) u_{0}}{y}\right) .
\end{aligned}
$$

Consequently $u_{0}=u(x, y)$. By (II), $u$ is continuous.

From (III), there exists a sequence $\left\{g_{n}\right\}$ of continuous complex-valued functions defined on $R$ such that $g_{n}(x) \rightarrow \psi(x)$ as $n \rightarrow \infty$ for each $x \in A$. For $n \geqq 2$, define

$$
h_{0}(x, y)=(y n(n+1)-n) g_{n}(u(x, y))+((n+1)-y n(n+1)) g_{n+1}(u(x, y))
$$

when $1 /(n+1) \leqq y \leqq 1 / n$. Then $h_{0}$ is continuous on $H_{0}$. Let $\left\{s_{n}\right\}_{n=1}^{\infty}$ be all the elements of $N$, where $n \neq m$ implies $s_{n} \neq s_{m}$. Let

$$
\begin{array}{ll}
r_{n}=\inf _{x>s_{n}} f(x), \\
l_{n}=\sup _{x<s_{n}} f(x)=f\left(s_{n}\right), \\
z_{n}=\phi\left(s_{n}\right)-\psi\left(s_{n}\right) & \text { if } s_{n} \in E, \\
z_{n}=0 & \text { if } s_{n} \notin E .
\end{array}
$$

Notice that $r_{n}-l_{n}>0$. If $x$ and $y$ are real numbers, define $x \vee y=\max \{x, y\}$ and $x \wedge y=\min \{x, y\}$. For $\langle x, y\rangle \in H_{0}$, set

$$
\Delta_{n}(x, y)=[(1-n y) \vee 0]\left[\left(1-\frac{1}{r_{n}-l_{n}}\left|r_{n}+l_{n}-2 s_{n}+2 \frac{s_{n}-x}{y}\right|\right) \vee 0\right] z_{n} .
$$

Then $\Delta_{n}$ is continuous in $H_{0}$. Observe that $\Delta_{n}(x, y)=0$ when $y \geqq 1 / n$. Using this fact, it is easy to show that, if we set

$$
h_{1}=h_{0}+\sum_{n=1}^{\infty} \Delta_{n}
$$

then $h_{1}$ is defined and continuous on $H_{0}$. 
Let $p$ be any point of $A$. The line

$$
x=(f(p)-p) y+p
$$

passes through $\langle p, 0\rangle$, and, since $|f(p)-p|<2^{1 / 2}-1=\operatorname{ctn} \cdot \frac{3}{8} \pi$, the part of this line which lies in $H_{0}$ is contained in $s\left(p, 1, \frac{3}{8} \pi\right)$. We show that $h_{1}$ approaches $\psi(p)$ along this line. By substituting $(f(p)-p) y+p$ for $x$ in the expression for $\Delta_{n}(x, y)$, one obtains

$$
\begin{aligned}
\Delta_{n}(x, y)=[(1-n y) & \vee 0] \\
& \cdot\left[\left(1-\frac{1}{r_{n}-l_{n}}\left|r_{n}+l_{n}+2\left(\frac{1}{y}-1\right)\left(s_{n}-p\right)-2 f(p)\right|\right) \vee 0\right] z_{n} .
\end{aligned}
$$

If $p \leqq s_{n}$, then $f(p) \leqq l_{n}$, and one can verify directly that (8) vanishes. If $p>s_{n}$, then $f(p) \geqq r_{n}$, and again one can verify directly that (8) vanishes. Thus $\Delta_{n}(x, y)$ vanishes along that part of the line (7) which lies in $H$.

Solving (7) for $f(p)$, we find that, along the given line,

and hence

$$
f(p)=(x-(1-y) p) / y
$$

$$
p=f^{*}(f(p))=f^{*}((x-(1-y) p) / y) .
$$

Therefore (if $\left.0<y \leqq \frac{1}{2}\right) p=u(x, y)$. Hence, if $\langle x, y\rangle$ satisfies (7), $n \geqq 2$, and $1 /(n+1)$ $\leqq y \leqq 1 / n$, then

$$
h_{0}(x, y)=(y n(n+1)-n) g_{n}(p)+((n+1)-y n(n+1)) g_{n+1}(p),
$$

so that $h_{0}(x, y)$ lies on the line segment joining $g_{n}(p)$ to $g_{n+1}(p)$. It follows that $h_{0}(x, y)$ approaches $\psi(p)$ as $\langle x, y\rangle$ approaches $p$ along the line (7). Since each $\Delta_{n}$ vanishes on the part of this line lying in $H, h_{1}(x, y)$ also approaches $\psi(p)$ along this line.

Let $s_{m}$ be any point of $E$. The definition of $f$ shows that

$$
|f(x)-x| \leqq \sum_{s \in N} w(s)
$$

for all $x$, and from this it easily follows that

Hence

$$
\left|r_{m}-s_{m}\right| \leqq \sum_{s \in N} w(s), \quad\left|l_{m}-s_{m}\right| \leqq \sum_{s \in N} w(s) .
$$

$$
\left|\frac{r_{m}+l_{m}}{2}-s_{m}\right| \leqq \sum_{s \in N} w(s)<2^{1 / 2}-1=\operatorname{ctn} \frac{3}{8} \pi .
$$

So the part of the line

$$
x=\left(\frac{r_{m}+l_{m}}{2}-s_{m}\right) y+s_{m}
$$

that lies in $H_{0}$ is contained in $s\left(s_{m}, 1, \frac{3}{8} \pi\right)$. We show that $h_{1}$ approaches $\phi\left(s_{m}\right)$ as 
$z \rightarrow s_{m}$ along this line. Substituting the value of $x$ given by (9) into the expression for $\Delta_{n}$, we obtain

$$
\begin{aligned}
& \Delta_{n}(x, y)=[(1-n y)\vee 0] \\
& \cdot\left[\left(1-\frac{1}{r_{n}-l_{n}}\left|r_{n}-r_{m}+l_{n}-l_{m}+2\left(\frac{1}{y}-1\right)\left(s_{n}-s_{m}\right)\right|\right) \vee 0\right] z_{n} .
\end{aligned}
$$

If $s_{m}<s_{n}$, then $l_{m}<r_{m} \leqq l_{n}<r_{n}$, and one can verify that (10) vanishes. If $s_{n}<s_{m}$, then $l_{n}<r_{n} \leqq l_{m}<r_{m}$, and again one can verify that (10) vanishes. Thus, for $n \neq m$, $\Delta_{n}(x, y)=0$ when $\langle x, y\rangle$ lies on the line (9) and in $H$.

If we take $n=m$ in (10), we obtain

$$
\Delta_{m}(x, y)=[(1-m y) \vee 0] z_{m} .
$$

Therefore $\Delta_{m}(x, y)$ approaches $z_{m}=\phi\left(s_{m}\right)-\psi\left(s_{m}\right)$ along the given line.

Take any $\langle x, y\rangle \in H_{0}$ satisfying (9), and take any $a$ and $b$ satisfying

$$
a<s_{m}<b .
$$

Then $f(a) \leqq l_{m}<\frac{1}{2}\left(r_{m}+l_{m}\right)<r_{m} \leqq f(b)$, so that

$$
\left(f(a)-s_{m}\right) y+s_{m}<x<\left(f(b)-s_{m}\right) y+s_{m} ;
$$

from which we deduce that

$$
f(a)<\left(x-(1-y) s_{m}\right) / y<f(b) .
$$

Since $f^{*}$ is weakly increasing,

$$
a=f^{*}(f(a)) \leqq f^{*}\left(\left(x-(1-y) s_{m}\right) / y\right) \leqq f^{*}(f(b))=b .
$$

Because $a$ and $b$ were taken to be any two numbers satisfying (11), we conclude that

$$
s_{m}=f^{*}\left(\left(x-(1-y) s_{m}\right) / y\right)
$$

whence it follows that $u(x, y)=s_{m}$. Thus

$$
h_{0}(x, y)=(y n(n+1)-n) g_{n}\left(s_{m}\right)+((n+1)-y n(n+1)) g_{n+1}\left(s_{m}\right)
$$

when $1 /(n+1) \leqq y \leqq 1 / n$. Consequently $h_{0}(x, y)$ approaches $\psi\left(s_{m}\right)$ along the line (9); so $h_{1}(x, y)$ approaches $\psi\left(s_{m}\right)+\phi\left(s_{m}\right)-\psi\left(s_{m}\right)=\phi\left(s_{m}\right)$ along the given line.

We have shown that, for each $x \in A$, there exists a line segment at $x$, lying in $s\left(x, 1, \frac{3}{8} \pi\right)$, such that $h_{1}(z) \rightarrow \phi(x)$ as $z \rightarrow x$ along the line segment. We do not know that $h_{1}$ is bounded, but this is easily patched up. Choose a real number $B$ such that, for all $x \in A$,

$$
-B<\operatorname{Re} \phi(x)<B, \quad-B<\operatorname{Im} \phi(x)<B
$$

and set

$$
h(z)=\left(\left[\left(\operatorname{Re} h_{1}(z)\right) \vee(-B)\right] \wedge B\right)+i\left(\left[\left(\operatorname{Im} h_{1}(z)\right) \vee(-B)\right] \wedge B\right) .
$$


If we extend $h$ to a bounded continuous function defined in $H$, and then restrict $h$ to $Q$, we have the desired function.

(C) Let $d(t)$ be a weakly increasing, positive, real-valued function defined for $0<t \leqq 1$. Then there exists a continuous, complex-valued function $k$ defined in $Q$, with $|k(z)| \leqq 2^{1 / 2}$ for all $z \in Q$, such that for each $a \in(0,1]$ and for each arc

$$
\gamma \subseteq\{\langle x, y\rangle:-1 \leqq x \leqq 1,0<y \leqq a\},
$$

(diameter $\gamma) \geqq d(a)$ implies $($ diameter $k(\gamma)) \geqq 2$.

Proof. Let $p(x)=\frac{1}{2} \int_{0}^{x} d(t) d t(0<x \leqq 1)$. Then $p$ is positive, continuous, and strictly increasing, and $p(x) \leqq \frac{1}{2} d(x)$. Let $a \in(0,1]$ be given. Since $p(x)^{-1}$ is uniformly continuous on each compact subset of $(0,1]$, there exists $\varepsilon \in(0,1]$ such that

$$
\left(\frac{1}{2} a \leqq x_{1} \leqq 1 \text { and }\left|x_{1}-x_{2}\right|<\varepsilon\right)
$$

implies

$$
\left|p\left(x_{1}\right)^{-1}-p\left(x_{2}\right)^{-1}\right| \leqq \frac{1}{2} .
$$

Let $\varepsilon(a)$ be the supremum of all such $\varepsilon$. Then $\varepsilon(a)$ is an increasing function of $a$, and

$$
\left(\frac{1}{2} a \leqq x_{1} \leqq 1 \text { and }\left|x_{1}-x_{2}\right|<\varepsilon(a)\right)
$$

implies

$$
\left|p\left(x_{1}\right)^{-1}-p\left(x_{2}\right)^{-1}\right| \leqq \frac{1}{2} .
$$

Set $q(x)=\int_{0}^{x} \varepsilon(t) d t$. Then $q$ is positive, continuous, and strictly increasing, and $q(x) \leqq \varepsilon(x)$. Let $m(x)=\min \{p(x), q(x)\}$. For $\langle x, y\rangle \in Q$, define

$$
\begin{gathered}
k_{1}(y)=\sin (2 \pi / y m(y)), \quad k_{2}(x, y)=\sin (4 \pi x / p(y)), \\
k(x, y)=k_{1}(y)+i k_{2}(x, y) .
\end{gathered}
$$

Now suppose that $a \in(0,1]$ is given, and suppose that $\gamma \subseteq\{\langle x, y\rangle:-1 \leqq x \leqq 1$, $0<y \leqq a\}$ is an arc with (diameter $\gamma) \geqq d(a)$. Choose $z_{1}=\left\langle x_{1}, y_{1}\right\rangle$ and $z_{2}=\left\langle x_{2}, y_{2}\right\rangle$ in $\gamma$ so that $\left|z_{1}-z_{2}\right| \geqq d(a)$. Assume without loss of generality that $y_{2} \leqq y_{1}$. We can choose $a^{\prime}$ so that $0<\frac{1}{2} a^{\prime} \leqq y_{1} \leqq a^{\prime} \leqq a$. Since $m\left(a^{\prime}\right) \leqq \frac{1}{2} d\left(a^{\prime}\right) \leqq \frac{1}{2} d(a)$, and since $\left|z_{1}-z_{2}\right| \geqq d(a)$, we must have either

$$
\left|y_{1}-y_{2}\right| \geqq m\left(a^{\prime}\right)
$$

or

$$
\left|y_{1}-y_{2}\right|<m\left(a^{\prime}\right) \text { and }\left|x_{1}-x_{2}\right| \geqq \frac{1}{2} d\left(a^{\prime}\right) .
$$

First assume that (12) holds. Here $m\left(y_{2}\right) \leqq m\left(y_{1}\right) \leqq m\left(a^{\prime}\right)$, so

$$
2 \pi / y_{1} m\left(y_{1}\right) \leqq 2 \pi / y_{2} m\left(y_{2}\right),
$$


and we have

$$
\begin{aligned}
\frac{2 \pi}{y_{2} m\left(y_{2}\right)}-\frac{2 \pi}{y_{1} m\left(y_{1}\right)} & =\frac{2 \pi\left(y_{1} m\left(y_{1}\right)-y_{2} m\left(y_{2}\right)\right)}{y_{1} y_{2} m\left(y_{1}\right) m\left(y_{2}\right)} \\
& \geqq \frac{2 \pi\left(y_{1} m\left(y_{2}\right)-y_{2} m\left(y_{2}\right)\right)}{y_{1} y_{2} m\left(y_{1}\right) m\left(y_{2}\right)}=\frac{2 \pi\left(y_{1}-y_{2}\right)}{y_{1} y_{2} m\left(y_{1}\right)} \\
& \geqq \frac{2 \pi m\left(a^{\prime}\right)}{y_{1} y_{2} m\left(y_{1}\right)} \geqq \frac{2 \pi}{y_{1} y_{2}} \geqq 2 \pi
\end{aligned}
$$

Thus, as $\langle x, y\rangle$ moves along $\gamma$ from $\left\langle x_{1}, y_{1}\right\rangle$ to $\left\langle x_{2}, y_{2}\right\rangle$, we see that $2 \pi / y m(y)$ varies over an interval of length at least $2 \pi$, and hence $k_{1}(y)$ varies over the whole of the interval $[-1,1]$. Therefore (diameter $k(\gamma)) \geqq 2$.

Now assume that (13) holds. Then

$$
\begin{aligned}
\left|\frac{4 \pi x_{1}}{p\left(y_{1}\right)}-\frac{4 \pi x_{2}}{p\left(y_{2}\right)}\right| & \geqq 4 \pi\left[\left|\frac{x_{1}}{p\left(y_{1}\right)}-\frac{x_{2}}{p\left(y_{1}\right)}\right|-\left|\frac{x_{2}}{p\left(y_{2}\right)}-\frac{x_{2}}{p\left(y_{1}\right)}\right|\right] \\
& \geqq 4 \pi\left[\frac{\left|x_{1}-x_{2}\right|}{p\left(y_{1}\right)}-\left|\frac{1}{p\left(y_{2}\right)}-\frac{1}{p\left(y_{1}\right)}\right|\right] \\
& \geqq 4 \pi\left[\frac{\frac{1}{2} d\left(a^{\prime}\right)}{p\left(a^{\prime}\right)}-\left|\frac{1}{p\left(y_{2}\right)}-\frac{1}{p\left(y_{1}\right)}\right|\right] \\
& \geqq 4 \pi\left[1-\left|\frac{1}{p\left(y_{2}\right)}-\frac{1}{p\left(y_{1}\right)}\right|\right] .
\end{aligned}
$$

Now, $\left|y_{1}-y_{2}\right|<m\left(a^{\prime}\right) \leqq q\left(a^{\prime}\right) \leqq \varepsilon\left(a^{\prime}\right)$, so $\left|p\left(y_{2}\right)^{-1}-p\left(y_{1}\right)^{-1}\right| \leqq \frac{1}{2}$. Therefore

$$
\left|4 \pi x_{1} / p\left(y_{1}\right)-4 \pi x_{2} / p\left(y_{2}\right)\right| \geqq 2 \pi,
$$

and we see that as $\langle x, y\rangle$ varies along $\gamma$ from $\left\langle x_{1}, y_{1}\right\rangle$ to $\left\langle x_{2}, y_{2}\right\rangle$, the quantity $4 \pi x / p(y)$ varies over an interval of length at least $2 \pi$, so that $k_{2}(x, y)$ takes on every value in the interval $[-1,1]$. Thus (diameter $k(\gamma)) \geqq 2$.

(D) Let $A \subseteq X$ be a set of type $F_{\sigma \delta}$, and let $\phi$ be a bounded function of honorary Baire class $\leqq 2\left(A, R^{2}\right)$. Then there exists a bounded continuous complex-valued function $f$ defined in $Q$ such that $A$ is the set of curvilinear convergence of $f$ and $\phi$ is a boundary function for $f$.

Proof. Let $g$ be the function of (A) and let $h$ be the function of (B). For $t \in(0,1]$, let

$$
\begin{gathered}
d_{1}(t)=\sup \left\{\delta \in(0,1]:\left(y_{1} \geqq t, y_{2} \geqq t,\left\langle x_{1}, y_{1}\right\rangle \in Q,\left\langle x_{2}, y_{2}\right\rangle \in Q,\right. \text { and }\right. \\
\left.\left.\left|\left\langle x_{1}, y_{1}\right\rangle-\left\langle x_{2}, y_{2}\right\rangle\right|<\delta\right) \text { implies }\left|h\left(x_{1}, y_{1}\right)-h\left(x_{2}, y_{2}\right)\right| \leqq t\right\}, \\
d_{2}(t)=\sup \left\{\delta \in(0,1]:\left(y_{1} \geqq t, y_{2} \geqq t,\left\langle x_{1}, y_{1}\right\rangle \in Q,\left\langle x_{2}, y_{2}\right\rangle \in Q,\right. \text { and }\right. \\
\left.\left.\left|\left\langle x_{1}, y_{1}\right\rangle-\left\langle x_{2}, y_{2}\right\rangle\right|<\delta\right) \text { implies }\left|g\left(x_{1}, y_{1}\right)-g\left(x_{2}, y_{2}\right)\right| \leqq t\right\}, \\
d(t)=\min \left\{\frac{1}{3} d_{1}\left(\frac{1}{2} t\right), \frac{1}{3} d_{2}\left(\frac{1}{2} t\right), \frac{1}{4} t\right\} .
\end{gathered}
$$

Let $k$ be the function of (C) for this $d(t)$, and set $f(z)=h(z)+g(z) k(z)(z \in Q)$. We show that $f$ is the desired function. 
Suppose $x \in A$. Then there exists an $\operatorname{arc} \gamma$ at $x$, lying in $s\left(x, 1, \frac{3}{8} \pi\right)$, such that $h$ approaches $\phi(x)$ along $\gamma$. But $g(z)$ approaches 0 through $s\left(x, 1, \frac{3}{8} \pi\right)$ and $k$ is bounded, so $g(z) k(z)$ approaches 0 along $\gamma$. Hence $f(z)$ approaches $\phi(x)$ along $\gamma$. Thus $\phi$ is a boundary function for $f$, and $A$ is a subset of the set of curvilinear convergence of $f$. It only remains to show that if $x$ is a point of the set of curvilinear convergence of $f$, then $x \in A$. To show this, let $\gamma$ be an arc at $x$ along which $f$ approaches a limit. We may assume without loss of generality that $\gamma$ has an end point in $\{\langle x, 1\rangle:-1$ $\leqq x \leqq 1\}$. By the properties of $g$, it will be enough to show that $g$ approaches zero along $\gamma$. Assume that $g$ does not approach zero along $\gamma$. Then there exists $\varepsilon \in(0,1]$ and there exists a sequence $\left\{z_{n}\right\}$ such that $z_{n} \in \gamma-\{x\}, z_{n} \rightarrow x$ as $n \rightarrow \infty$, and $\left|g\left(z_{n}\right)\right| \geqq \varepsilon$ for all $n$. Write $z_{n}=\left\langle x_{n}, y_{n}\right\rangle$. Choose $N$ so that $n \geqq N$ implies $y_{n}<\frac{1}{4} \varepsilon$.

For the time being, let $n$ be a fixed integer greater than or equal to $N$. Set $a=4 y_{n} / 3$. Let $\gamma^{\prime}$ be the component of $\gamma \cap \mathrm{Cl}\left[S\left(d(a), z_{n}\right)\right]$ that contains $z_{n}$. (Recall that $S\left(d(a), z_{n}\right)=\left\{z:\left|z-z_{n}\right|<d(a)\right\}$.) Then

$$
d(a) \leqq \text { diameter } \gamma^{\prime} \leqq 2 d(a)
$$

and, since $d(a) \leqq \frac{1}{4} a$,

$$
\gamma^{\prime} \subseteq\left\{\langle x, y\rangle: \frac{1}{2} a \leqq y \leqq a\right\} .
$$

By the choice of $k$, there exist points $p$ and $q$ in $\gamma^{\prime}$ with $|k(p)-k(q)| \geqq 2$. We have $|p-q| \leqq 2 d(a)<d_{1}\left(\frac{1}{2} a\right)$, so, by the definition of $d_{1}(t)$,

Similarly,

$$
|h(p)-h(q)| \leqq \frac{1}{2} a<\frac{1}{6} \varepsilon
$$

$$
\begin{aligned}
\left|g(p)-g\left(z_{n}\right)\right| & \leqq \frac{1}{2} a<\frac{1}{6} \varepsilon \\
\left|g(q)-g\left(z_{n}\right)\right| & \leqq \frac{1}{2} a<\frac{1}{6} \varepsilon .
\end{aligned}
$$

Thus

$$
\begin{aligned}
|f(p)-f(q)| \geqq & |g(p) k(p)-g(q) k(q)|-|h(p)-h(q)| \\
> & \mid g(p) k(p)-g\left(z_{n}\right) k(p)+g\left(z_{n}\right) k(p)-g\left(z_{n}\right) k(q) \\
\geqq & \left|g\left(z_{n}\right)\right||k(p)-k(q)|-|k(p)|\left|g(p)-g\left(z_{n}\right)\right| \\
& \quad-|k(q)|\left|g(q)-g\left(z_{n}\right)\right|-\frac{1}{6} \varepsilon \quad \\
\geqq & 2 \varepsilon-2^{1 / 2} \frac{1}{6} \varepsilon-2^{1 / 2} \frac{1}{6} \varepsilon-\frac{1}{6} \varepsilon>\varepsilon .
\end{aligned}
$$

Note that $\left|p-z_{n}\right| \leqq d(a) \leqq \frac{1}{4} a=\frac{1}{3} y_{n}$, and similarly $\left|q-z_{n}\right| \leqq \frac{1}{3} y_{n}$.

We have now shown that, for each $n \geqq N$, there exist points $p_{n}, q_{n} \in \gamma$ with $\left|p_{n}-z_{n}\right| \leqq \frac{1}{3} y_{n},\left|q_{n}-z_{n}\right| \leqq \frac{1}{3} y_{n}$, and $\left|f\left(p_{n}\right)-f\left(q_{n}\right)\right|>\varepsilon$. But then $p_{n} \rightarrow x$ and $q_{n} \rightarrow x$ as $n \rightarrow \infty$, so $f$ does not approach a limit along $\gamma$. This is a contradiction. We conclude that $g(z) \rightarrow 0$ along $\gamma$, and hence that $x \in A$.

(E) Let $A \subseteq C$ be a set of type $F_{\sigma \delta}$, and let $\phi$ be a bounded function of honorary Baire class $\leqq 2\left(A, R^{2}\right)$. Then there exists a bounded continuous complex-valued function $f$ defined in $D$ such that $A$ is the set of curvilinear convergence of $f$ and $\phi$ is a boundary function for $f$. 
Proof. If $A=\varnothing$, this is trivial. If $A \neq \varnothing$, then we can assume, by making a suitable rotation of the disk, that $\langle 1,0\rangle \in A$. Let $G=D-S\left(\frac{1}{2},\left\langle\frac{1}{2}, 0\right\rangle\right)$ and let $L=C$ $-\{\langle 1,0\rangle\}$. Because $Q \cup X$ is homeomorphic with $G \cup L$, we see from (D) that there exists a bounded continuous complex-valued function $f_{1}$ defined in $G$ such that

(i) $A \cap L$ is the set of all points $x \in L$ such that $f_{1}$ approaches a limit along some arc at $x$, and

(ii) the restriction of $\phi$ to $L$ is a boundary function for $f_{1}$.

Since $G$ is closed relative to $D$, we can extend $f_{1}$ to a bounded continuous function $f$ defined in $D$ in such a way that $f$ has $\phi(\langle 1,0\rangle)$ as a radial limit at $\langle 1,0\rangle$. This $f$ will have all the desired properties.

(F) Let $S^{2}$ denote the Riemann sphere, let $A \subseteq C$ be a set of type $F_{\sigma o}$, and let $\phi$ be a function of honorary Baire class $\leqq 2\left(A, S^{2}\right)$. Then there exists a continuous function $f: D \rightarrow S^{2}$ such that $A$ is the set of curvilinear convergence of $f$ and $\phi$ is a boundary function for $f$.

(I) We suppose that

$$
S^{2}=\left\{\langle x, y, z\rangle \in R^{3}: x^{2}+y^{2}+z^{2}=1\right\} .
$$

We let

$$
\begin{aligned}
U & =\left\{\langle x, y, z\rangle \in S^{2}:-\frac{1}{2^{1 / 2}}<z \leqq 1\right\}, \\
V & =\left\{\langle x, y, z\rangle \in S^{2}:-1 \leqq z<\frac{1}{2^{1 / 2}}\right\}, \\
Z_{U} & =\left\{\langle x, y, z\rangle \in S^{2}: \frac{1}{2^{1 / 2}}<z \leqq 1\right\}, \\
Z_{V} & =\left\{\langle x, y, z\rangle \in S^{2}:-1 \leqq z<-\frac{1}{2^{1 / 2}}\right\}
\end{aligned}
$$

We define mappings $\Phi_{U}: Z_{U} \rightarrow U$ and $\Phi_{V}: Z_{V} \rightarrow V$ by setting

$$
\Phi_{U}(x, y, z)=\left\langle x\left(4 z^{2}-1\right), y\left(4 z^{2}-1\right), z\left(4 z^{2}-3\right)\right\rangle \quad\left(\langle x, y, z\rangle \in Z_{U}\right)
$$

and

$$
\Phi_{V}(x, y, z)=\left\langle x\left(4 z^{2}-1\right), y\left(4 z^{2}-1\right), z\left(4 z^{2}-3\right)\right\rangle \quad\left(\langle x, y, z\rangle \in Z_{V}\right) .
$$

Then $\Phi_{U}$ is a one-to-one continuous function from $Z_{U}$ onto $U$. Since $Z_{U}$ and $U$ are each homeomorphic to the unit disk $D$, it follows from [7, Corollary 1, p. 122] that $\Phi_{U}$ is a homeomorphism of $Z_{U}$ onto $U$. Similarly, $\Phi_{V}$ is a homeomorphism of $Z_{V}$ onto $V$.

We define a continuous function $\Phi: S^{2} \rightarrow S^{2}$ by setting

$$
\begin{array}{ll}
\Phi(x, y, z)=\Phi_{U}(x, y, z), & \frac{1}{2^{1 / 2}}<z \leqq 1, \\
\Phi(x, y, z)=\langle x, y,-z\rangle, & -\frac{1}{2^{1 / 2}} \leqq z \leqq \frac{1}{2^{1 / 2}}, \\
\Phi(x, y, z)=\Phi_{V}(x, y, z), & -1 \leqq z<-\frac{1}{2^{1 / 2}} .
\end{array}
$$


Notice that for each $p \in S^{2}$, the inverse image set $\Phi^{-1}(\{p\})$ contains at most three points.

(II) Most of the results of Hausdorff [2] on real-valued Baire functions can easily be shown to hold also for functions taking values in $R^{n}$. We shall make free use of these results in this more general form.

(III) Now we proceed to the proof of (F). Let $N$ be a countable subset of $A$ such that the restriction of $\phi$ to $A-N$ is of Baire class $\leqq 1\left(A-N, S^{2}\right)$, and let $A_{1}=A-N$. It will be convenient to let $F_{\sigma}\left(A_{1}\right)$ denote the class of all subsets of $A_{1}$ that are of type $F_{\sigma}$ relative to $A_{1}$, and $G_{\delta}\left(A_{1}\right)$ the class of all subsets of $A_{1}$ that are of type $G_{\delta}$ relative to $A_{1}$. Since $U$ and $V$ are open subsets of $S^{2}$ and $U \cup V=S^{2}$, we see that $A_{1} \cap \phi^{-1}(U) \in F_{\sigma}\left(A_{1}\right), A_{1}-\phi^{-1}(V) \in G_{\delta}\left(A_{1}\right)$, and $A_{1}-\phi^{-1}(V) \subseteq A_{1}$ $\cap \phi^{-1}(U)$. An elegant theorem of Sierpinski [8] now enables us to choose a set $K \in F_{\sigma}\left(A_{1}\right) \cap G_{\delta}\left(A_{1}\right)$ such that

$$
A_{1}-\phi^{-1}(V) \subseteq K \subseteq A_{1} \cap \phi^{-1}(U) .
$$

Let $L=A_{1}-K$. Then $L \in F_{\sigma}\left(A_{1}\right) \cap G_{\delta}\left(A_{1}\right)$. Moreover, $\phi(K) \subseteq U$ and $\phi(L) \subseteq V$.

Let $p_{1}=\langle 1,0,0\rangle$, and define $\psi: A \rightarrow S^{2}-\left\{p_{1}\right\}$ as follows. Set

$$
\begin{array}{ll}
\psi(x)=\Phi_{U}^{-1}(\phi(x)), & x \in K, \\
\psi(x)=\Phi_{V}^{-1}(\phi(x)), & x \in L .
\end{array}
$$

If $x \in N$, we let $\psi(x)$ be any element of $Z_{U} \cup Z_{V}$ for which $\Phi(\psi(x))=\phi(x)$. This choice of $\psi(x)$ is always possible, because $\Phi\left(Z_{U} \cup Z_{V}\right) \supseteq U \cup V=S^{2}$. Let $\psi_{0}$ be the restriction of $\psi$ to $A_{1}=K \cup L$. I assert that $\psi_{0}$ is of Baire class $\leqq 1\left(A_{1}, S^{2}-\left\{p_{1}\right\}\right)$. Since $S^{2}-\left\{p_{1}\right\}$ is homeomorphic to $R^{2}$, it will suffice to show that $\psi_{0}^{-1}(G) \in F_{\sigma}\left(A_{1}\right)$ for every open set $G \subseteq S^{2}-\left\{p_{1}\right\}$. But

$$
\begin{aligned}
\psi_{0}^{-1}(G) & =A_{1} \cap \psi^{-1}(G)=\left[K \cap \psi^{-1}(G)\right] \cup\left[L \cap \psi^{-1}(G)\right] \\
& =\left[K \cap \phi^{-1}\left(\Phi_{U}\left(Z_{U} \cap G\right)\right)\right] \cup\left[L \cap \phi^{-1}\left(\Phi_{V}\left(Z_{V} \cap G\right)\right)\right] \in F_{\sigma}\left(A_{1}\right),
\end{aligned}
$$

so $\psi_{0}$ is of Baire class $\leqq 1\left(A_{1}, S^{2}-\left\{p_{1}\right\}\right)$. Now, $A_{1}=A-N$ is of type $G_{\delta}$ relative to $A$, so (again using the fact that $S^{2}-\left\{p_{1}\right\}$ is homeomorphic to $R^{2}$ ) we can extend $\psi_{0}$ to a function $\psi_{1}$ of Baire class $\leqq 1\left(A, S^{2}-\left\{p_{1}\right\}\right)$. The existence of $\psi_{1}$ shows that $\psi$ is of honorary Baire class $\leqq 2\left(A, S^{2}-\left\{p_{1}\right\}\right)$. The range of $\psi$ is contained in $Z_{U} \cup Z_{V}$, so that the values of $\psi$ are bounded away from $p_{1}$. Thus, if we still think of $S^{2}-\left\{p_{1}\right\}$ as corresponding to the plane $R^{2}, \psi$ corresponds to a bounded function. By (E), there exists a continuous function $f_{1}: D \rightarrow S^{2}-\left\{p_{1}\right\}$ such that the values of $f_{1}$ are bounded away from $p_{1}, A$ is the set of curvilinear convergence of $f_{1}$, and $\psi$ is a boundary function for $f_{1}$. Let $f$ denote the composite function $\Phi \circ f_{1}$. Then $f$ is continuous and $\Phi \circ \psi=\phi$ is a boundary function for $f$. It only remains to show that if $x$ is a point of the set of curvilinear convergence of $f$, then $x \in A$. Let $\gamma$ be an arc at $x$ along which $f$ approaches a limit, and let $C\left(f_{1}, \gamma\right)$ denote the cluster set of $f_{1}$ along $\gamma$. Assume that $x \notin A$. Then $f_{1}$ does not approach a limit along $\gamma$, 
so $C\left(f_{1}, \gamma\right)$ contains infinitely many points. Now, $\Phi$ maps at most three points to any one given point, so $\Phi\left(C\left(f_{1}, \gamma\right)\right)$ contains infinitely many points. But $\Phi\left(C\left(f_{1}, \gamma\right)\right)$ is the cluster set of $f=\Phi \circ f_{1}$ along $\gamma$, and hence $f$ does not approach a limit along $\gamma$, contrary to our assumption. We conclude that $x \in A$ after all. This completes the proof of the theorem.

The following questions remain open.

Problem 1. If $A$ is an arbitrary set of type $F_{\sigma \delta}$ in $C$, does there necessarily exist a continuous real-valued function in $D$ having $A$ as its set of curvilinear convergence?

Problem 2. If $A \subseteq C$ is a set of type $F_{\sigma \delta}$, and if $\phi$ is a function of honorary Baire class $\leqq 2(A, R)$, does there necessarily exist a continuous real-valued function in $D$ having $A$ as its set of curvilinear convergence and $\phi$ as a boundary function?

Appendix. Some theorems concerning functions of Baire class $\leqq 1$ which take values on the Riemann sphere can be obtained by the technique used to prove $(F)$. We use the notation set up in the proof of $(F)$.

TheOREM (a). Let $M$ be a metric space, and let $\phi: M \rightarrow S^{2}$ be a function such that $\phi^{-1}(G)$ is an $F_{\sigma}$ set for every open set $G \subseteq S^{2}$. Then $\phi$ is of Baire class $\leqq 1\left(M, S^{2}\right)$.

Proof. Since $U$ and $V$ are open and $U \cup V=S^{2}$, it follows that the set $\phi^{-1}(U)$ is $F_{\sigma}$, the set $M-\phi^{-1}(V)$ is $G_{\delta}$, and $M-\phi^{-1}(V) \subseteq \phi^{-1}(U)$. By the theorem of Sierpinski [8], there exists a set $K$ that is simultaneously $F_{\sigma}$ and $G_{\delta}$ such that

$$
M-\phi^{-1}(V) \subseteq K \subseteq \phi^{-1}(U) .
$$

Let $L=M-K$. Then $L$ is simultaneously $F_{\sigma}$ and $G_{\delta}$, and

$$
\phi(K) \subseteq U, \quad \phi(L) \subseteq V .
$$

Define $\psi: M \rightarrow S^{2}-\left\{p_{1}\right\}$ (where $p_{1}=\langle 1,0,0\rangle$ ) by setting

$$
\psi(x)=\Phi_{U}^{-1}(\phi(x)), \quad x \in K, \quad \psi(x)=\Phi_{V}^{-1}(\phi(x)), \quad x \in L .
$$

If $G$ is an open subset of $S^{2}-\left\{p_{1}\right\}$, then

$$
\psi^{-1}(G)=\left[K \cap \phi^{-1}\left(\Phi_{U}\left(Z_{U} \cap G\right)\right)\right] \cup\left[L \cap \phi^{-1}\left(\Phi_{V}\left(Z_{V} \cap G\right)\right)\right],
$$

so $\psi^{-1}(G)$ is an $F_{\sigma}$ set. Since $S^{2}-\left\{p_{1}\right\}$ is homeomorphic to the plane, it follows that there exists a sequence $\left\{\psi_{n}\right\}$ of continuous functions, each mapping $M$ into $S^{2}-\left\{p_{1}\right\}$, such that $\psi_{n} \rightarrow \psi$ pointwise on $M$. But then $\Phi\left(\psi_{n}(x)\right) \rightarrow \Phi(\psi(x))=\phi(x)$ for each fixed $x \in M$, so $\phi$ is of Baire class $\leqq 1\left(M, S^{2}\right)$.

A special case of Theorem (b) was proved (in effect) in [6, proof of Theorem 6] by means of a rather messy lemma (Lemma 3). Theorem (a) provides a proof that is both more general and more esthetically satisfactory.

TheOREM (b). Let $M$ be a metric space, and let $\phi: M \rightarrow S^{2}$ be a function. Then $\phi$ is of honorary Baire class $\leqq 2\left(M, S^{2}\right)$ if, and only if, there exists a countable set $N \subseteq M$ such that, for every closed set $F \subseteq S^{2}, \phi^{-1}(F)-N$ is a $G_{\delta}$ set. 
Proof. The implication in one direction is trivial. Now assume that $N$ is countable and that $\phi^{-1}(F)-N$ is a $G_{\delta}$ set for every closed set $F \subseteq S^{2}$. Let $\phi_{0}$ be the restriction of $\phi$ to $M-N$. Since $S^{2}$ is a subset of $R^{3}, \phi_{0}$ is of Baire class $\leqq 1\left(M-N, R^{3}\right)$. Because $M-N$ is a $G_{\delta}$ set, $\phi_{0}$ can be extended to a function $\phi_{1}$ of Baire class $\leqq 1\left(M, R^{3}\right)$. Now, $\phi_{1}(x) \in S^{2}$ except for only countably many $x$, so there exists some point $q$ in the open ball enclosed by $S^{2}$ such that $q$ is not in the range of $\phi_{1}$. Define a mapping $P: R^{3}-\{q\} \rightarrow S^{2}$ as follows. If $a \in R^{3}-\{q\}$, let $L$ be the ray with end point at $q$ which passes through $a$, and let $P(a)$ be the intersection point of $L$ with $S^{2}$. Then $P$ is continuous and $P(a)=a$ for each $a \in S^{2}$. Let $\psi=P \circ \phi_{1}$. If $G \subseteq S^{2}$ is open, then $\psi^{-1}(G)=\phi_{1}^{-1}\left(P^{-1}(G)\right)$, so that $\psi^{-1}(G)$ is an $F_{\sigma}$ set. Thus, by Theorem (a), $\psi$ is of Baire class $\leqq 1\left(M, S^{2}\right)$. Moreover, if $x \notin N$, then $\phi_{1}(x)=\phi_{0}(x)$ $=\phi(x) \in S^{2}$, so that $\psi(x)=P(\phi(x))=\phi(x)$. Therefore $\phi$ is of honorary Baire class $\leqq 2\left(M, S^{2}\right)$.

An alternative proof of Theorem (b) could be given by combining Theorem (a) with the following result.

Theorem (c). Let $M$ be a metric space, $E$ a $G_{\delta}$ set in $M, \phi$ a function of Baire class $\leqq 1\left(E, S^{2}\right)$. Then $\phi$ can be extended to a function of Baire class $\leqq 1\left(M, S^{2}\right)$.

To prove this, use the technique appearing in the proof of Theorem (a).

Finally, we note that a theorem proved by Bagemihl and McMillan for realvalued functions [1, Theorem 2] can be transferred to the Riemann sphere by means of our technique.

\section{REFERENCES}

1. F. Bagemihl and J. E. McMillan, Characterization of the sets of angular and global convergence, and of the sets of angular and global limits, of functions in a half-plane, Fund. Math. 59 (1966), 177-187.

2. F. Hausdorff, Uber halbstetige Funktionen und deren Verallgemeinerung, Math. Z. 5 (1919), 292-309.

3. T. J. Kaczynski, Boundary functions for functions defined in a disk, J. Math. Mech. 14 (1965), 589-612.

4. - On a boundary property of continuous functions, Michigan Math. J. 13 (1966), 313-320.

5. - Boundary functions, Doctoral dissertation, University of Michigan, Ann Arbor, Mich., 1967.

6. J. E. McMillan, Boundary properties of functions continuous in a disc, Michigan Math. J. 13 (1966), 299-312.

7. M. H. A. Newman, Elements of the topology of plane sets of points, 2nd ed., Cambridge Univ. Press, New York, 1964.

8. W. Sierpinski, Sur une propriété des ensembles ambigus, Fund. Math. 6 (1924), 1-5.

9. L. E. Snyder, Bi-arc boundary functions, Proc. Amer. Math. Soc. 18 (1967), 808-811.

UNIVERSITY OF MICHIGAN, ANN Arbor, Michigan

UNIVERSITY OF CALIFORNIA, BERKELEY, CALIFORNIA 